\title{
International Journal of
}

\section{Computer Network and Information Security}

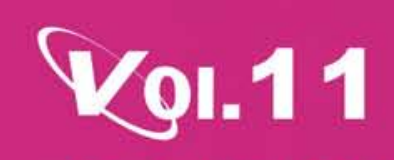

No.1 Jan. 2019

\section{IJCNIS Kol.11} Http:// www.mecs-press .org Vol. 11 No.1 January 2019
Modern Education and Computer Science PRESS 
International Journal of Computer Network and Information Security (IJCNIS)

ISSN Print: 2074-9090, ISSN Online: 2074-9104

Volume 11, Number 1, January 2019

\section{Contents}

\section{REGULAR PAPERS}

Identification of Compromised Nodes in MANETs using Machine Learning Technique

Rodney Sebopelo, Bassey Isong, Naison Gasela

A Comparative Study of Recent Steganography Techniques for Multiple Image Formats

Arshiya Sajid Ansari, Mohammad Sajid Mohammadi, Mohammad Tanvir Parvez

Understanding the Evolution of Ransomware: Paradigm Shifts in Attack Structures

Aaron Zimba, Mumbi Chishimba

Analyzing Progressive-BKZ Lattice Reduction Algorithm

Md. Mokammel Haque, Mohammad Obaidur Rahman

A Critical appraisal on Password based Authentication

Amanpreet A. Kaur, Khurram K. Mustafa

Cyber Security and Analysis of Cyber-Crime Laws to Restrict Cyber Crime in Pakistan 